\title{
Tortícolis como forma de presentación de quiste de la bolsa de Blake. Diagnóstico ecográfico en atención primaria
} Torticolis as a form of presentation of Blake's pouch cyst. Ultrasound diagnosis in primary care

\author{
Dr. Luis Ortiz González ${ }^{a *}$, Estud. Luis Ortiz Peces ${ }^{b}$ y Estud. Carlos Ortiz Peces. ${ }^{c}$
}

\section{RESUMEN}

La tortícolis es un signo clínico definido por la inclinación lateral del cuello y rotación de la cabeza, que puede ser fija o flexible y acompañarse o no de dolor cervical. Aparece en trastornos de diferente complejidad. Ante un caso de tortícolis, es preciso realizar una historia clínica cuidadosa y un examen físico completo, $y$, en caso de ser persistente, solicitar pruebas de imagen.

Se hace referencia a una causa de tortícolis no descrita en la literatura. Se trata de una tumoración quística compresiva en la fosa craneal posterior, quiste de la bolsa de Blake, en una lactante pequeña diagnosticada mediante ecografía en la consulta de Pediatría de Atención Primaria. Tras el diagnóstico, se remitió al centro hospitalario de referencia, donde se intervino de urgencia por Neurocirugía Pediátrica, mediante fenestración de la tumoración por ventriculostomía endoscópica y derivación ventrículo-peritoneal. Actualmente, se encuentra asintomática y sin secuelas.

Palabras clave: tortícolis, pediatría, quiste de la bolsa de Blake, ultrasonografía, atención primaria de salud.

\begin{abstract}
Torticollis is a clinical sign defined by the lateral inclination of the neck and rotation of the head, which can be fixed or flexible and accompanied or not by cervical pain. It appears in disorders of different complexity. In a case of torticollis it is necessary to carry out a careful medical history and a complete physical examination and, if persistent, request imaging tests. Reference is made to a cause of torticollis not described in the literature. This is a compressive cystic tumor in the posterior cranial fossa, Blake's pouch cyst, in a small infant diagnosed by ultrasound in the Primary Care Pediatrics office. After diagnosis, she was referred to the referral hospital, where emergency intervention was performed by pediatric neurosurgery, by fenestration of the tumor by endoscopic ventriculostomy and ventriculo-peritoneal shunt. She is currently asymptomatic and without sequelae.

Keywords: torticollis, pediatrics, Blake's pouch cyst, ultrasonography, primary health care.
\end{abstract}

a. Departamento de Ciencias Biomédicas, Facultad de Medicina, Universidad de Extremadura.

b. Facultad de Medicina, Universidad Complutense de Madrid, Pza. Ramón y Cajal, s/n, Ciudad Universitaria.

c. Facultad de Medicina, Universidad de Lleida, Lleida. España.

Correspondencia: Dr. Luis Ortiz González: lortiz@unex.es

Financiamiento: Ninguno.

Conflicto de intereses: Ninguno que declarar.

Recibido: 15-2-2020

Aceptado: 15-4-2020 http:/ / dx.doi.org/10.5546/ aap.2020.e495

Cómo citar: Ortiz González L, Ortiz Peces L, Ortiz Peces C. Tortícolis como forma de presentación de quiste de la bolsa de Blake. Diagnóstico ecográfico en atención primaria. Arch Argent Pediatr 2020;118(5):e495-e498.

\section{INTRODUCCIÓN}

En la atención inicial de un lactante con tortícolis, con buen estado general y desarrollo psicomotor normal, antes de emitir el diagnóstico clínico de tortícolis benigna del lactante, se deben considerar otros tipos de procesos etiológicos de mayor complejidad. Desde un punto de vista teórico, se distingue entre tortícolis congénitas y adquiridas, y si son dolorosas o no. ${ }^{1}$ En la práctica clínica ordinaria, este tema es mucho más amplio.

La más frecuente de ellas es la tortícolis muscular congénita, que, con una incidencia estimada del 0,3-1,9\%, ${ }^{1}$ suele manifestarse clínicamente a partir de la tercera semana de vida. También hay otros tipos de malformaciones congénitas que debutan clínicamente con posterioridad. La evaluación de si se trata de un proceso álgido o no también es cuestionable en esta época de la vida.

En pediatría, la prevalencia de la tortícolis de etiología muscular asciende casi a un $80 \%$ de los casos. ${ }^{2}$ En el resto de pacientes, hay que descartar anomalías óseas (síndrome de KlippelFeil) y patologías neurológica, ocular, digestiva (síndrome de Sandifer), traumática e infecciosa. Entre los procesos neurológicos, se destacan los tumores de la columna cervical superior $\mathrm{y}$ fosa craneal posterior (FCP); estos últimos, mayormente, en tortícolis intermitente. ${ }^{3}$ En estos casos, las pruebas de imagen son determinantes para el diagnóstico. En este sentido, en la Atención Primaria (AP) de Salud, la ecografía clínica, también denominada ecografía a pie de cama, ${ }^{4}$ puede llegar a ser muy útil en el manejo de este tipo de procesos. ${ }^{5}$ 
Se presenta un caso de tortícolis asociado a un quiste de la bolsa de Blake (QBB) en una lactante pequeña diagnosticada mediante ecografía en la consulta de Pediatría de AP.

\section{CASO CLÍNICO}

Se trata de una lactante, de 3 meses de edad, que presentaba un cuadro de una semana de evolución caracterizado por tortícolis intermitente. Antes de acudir por este motivo a nuestro Centro, había sido evaluada en 2 ocasiones en un servicio de urgencias hospitalario, donde se hizo el diagnóstico y se trató como otitis media aguda derecha.

Era la segunda hija de padres sanos, no consanguíneos, sin abortos previos y sin antecedentes familiares de interés clínico. La hermana, de 4 años de edad, sana. Embarazo controlado y bien tolerado, en cuyos controles ecográficos prenatales no se detectaron anomalías. Parto eutócico a las 38 semanas de gestación. Peso al nacer: 3,040 kg. Talla: $49 \mathrm{~cm}$. Perímetro cefálico: $34 \mathrm{~cm}$. Apgar: 10/10. Reanimación tipo I. Período neonatal: normal. Lactancia mixta desde el nacimiento. Desarrollo psicomotor normal con sonrisa social al mes de vida y sostén cefálico adquirido a los meses de edad. Inmunizaciones adecuadas y sin reacciones adversas relevantes. Detección precoz de alteraciones endocrinometabólicas y otoemisiones acústicas sin alteraciones.

En la exploración física, no se observaron otras alteraciones (Figura 1) diferentes a la lateralización derecha de la extremidad cefálica y discreto grado de irritabilidad. Se realizó una ecografía cerebral en la que se observó, en el corte coronal, una hidrocefalia triventricular con marcada dilatación de astas frontales y una formación quística en la FCP (hidrocefalia tetraventricular), centrada en la línea media (Figura 2). En la sección sagital, aparentaba comprimir anteriormente el $4^{\circ}$ ventrículo y se cuantificó su tamaño ( $35 \mathrm{x}$ $42 \mathrm{~mm}$ ) (Figura 4).

Ante la sospecha de QBB, se remitió al centro hospitalario de referencia, donde se intervino de urgencia por Neurocirugía Pediátrica, mediante fenestración de la tumoración por ventriculostomía endoscópica y derivación ventrículo-peritoneal. Tras la cirugía, la paciente ha sido controlada en nuestro Centro, donde se han realizado controles ecográficos transcraneales periódicos a través de la escama temporal con especial atención a los índices ventricular de Levene y de resistencia de Pourcelot (Figura 4). Actualmente, se encuentra asintomática y sin secuelas.

Figura 1. Evolución del perímetro craneal en los dos primeros años de edad

\section{Perímetro cefálico}

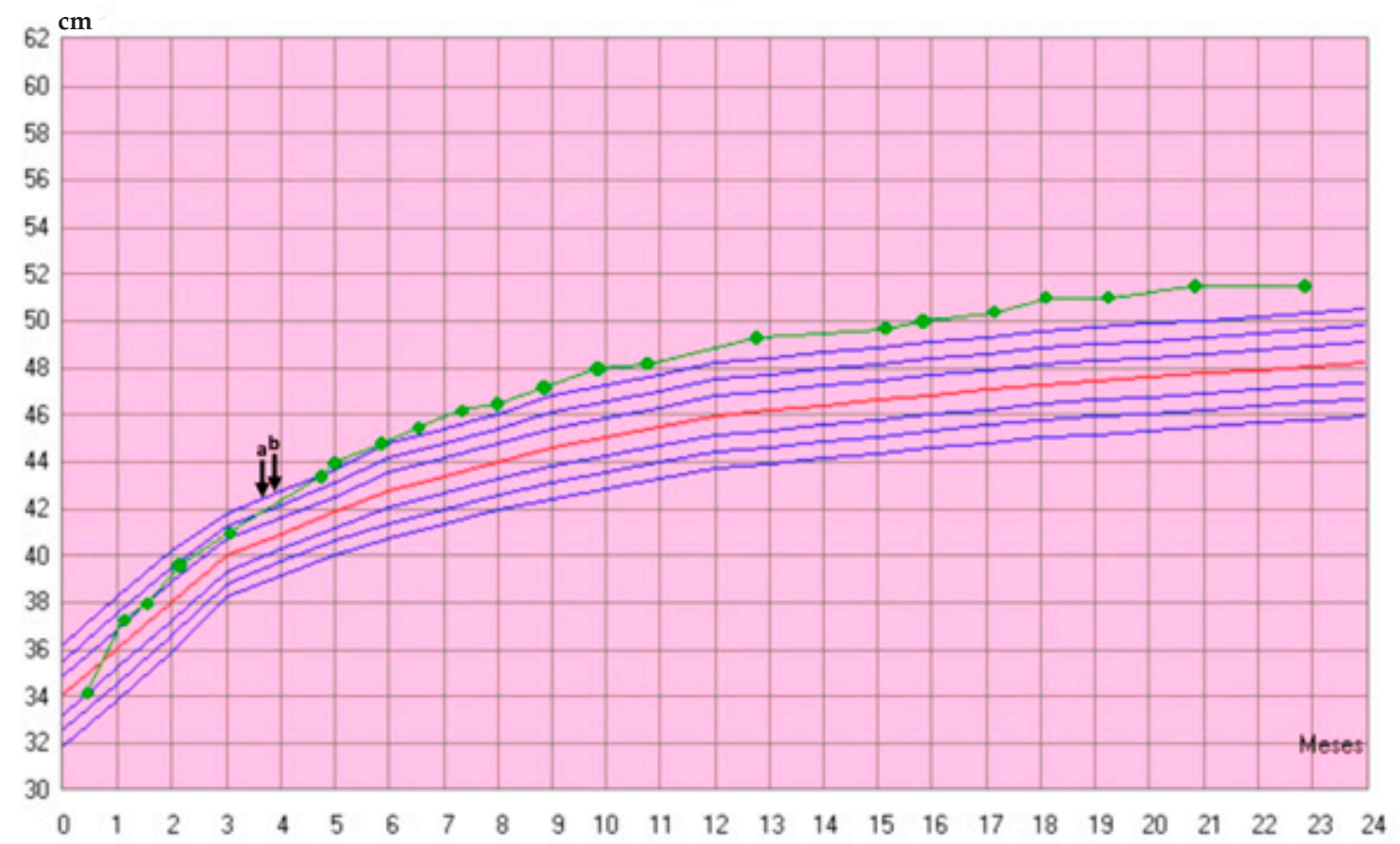

Las flechas indican el inicio de la clínica (a) y la cirugía (b). 
FIGURA 2. Visión frontal de corte coronal a nivel del agujero de Monro. Se observa la marcada dilatación de las astas frontales y temporales de los ventrículos laterales y del III ventrículo. Véase la compresión anterior que ejerce el quiste de la bolsa de Blake sobre la pared posterior del IV ventrículo

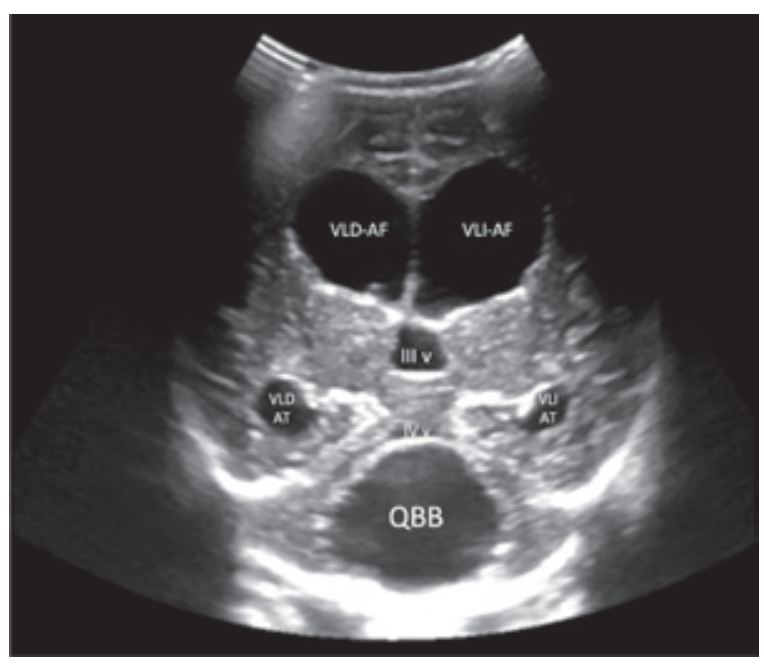

VLD-AF: asta frontal del ventrículo lateral derecho; VLI-AF: asta frontal del ventrículo lateral izquierdo; III v: tercer ventrículo;

VLD AT: asta temporal del ventrículo lateral derecho; VLI AT: asta temporal del ventrículo lateral izquierdo; IV v: cuarto ventrículo;

QBB: quiste de la bolsa de Blake.
FIGURA 3. Visión lateral de corte sagital a nivel de la línea media en el que se cuantifica el tamaño bidimensional del quiste de la bolsa de Blake. Se observa la dilatación del asta frontal del ventrículo lateral y del III ventrículo. Se muestran también el agujero de Monro y el acueducto de Silvio

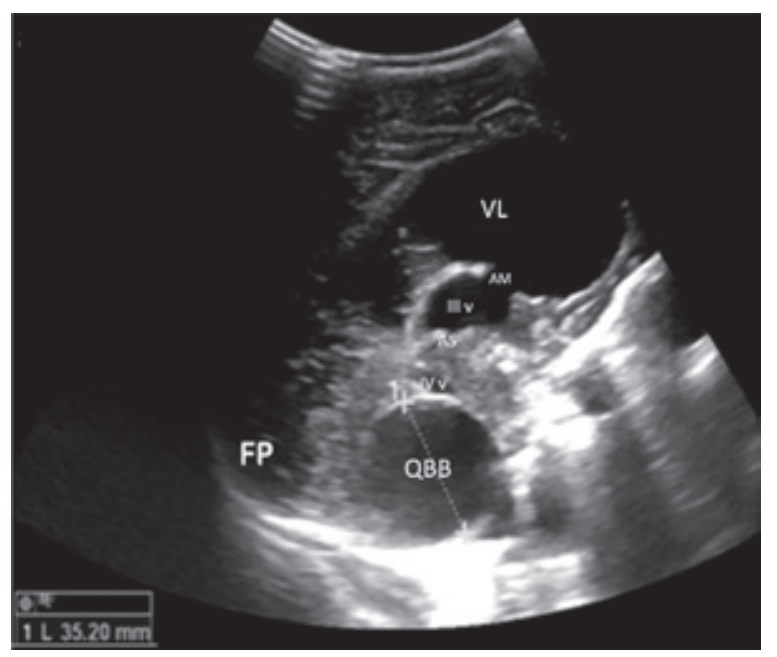

VL: asta frontal del ventrículo lateral;

AM: agujero de Monro; I

II v: tercer ventrículo;

AS: acueducto de Silvio;

IV v: cuarto ventrículo;

QBB: quiste de la bolsa de Blake;

FP: fosa craneal posterior.

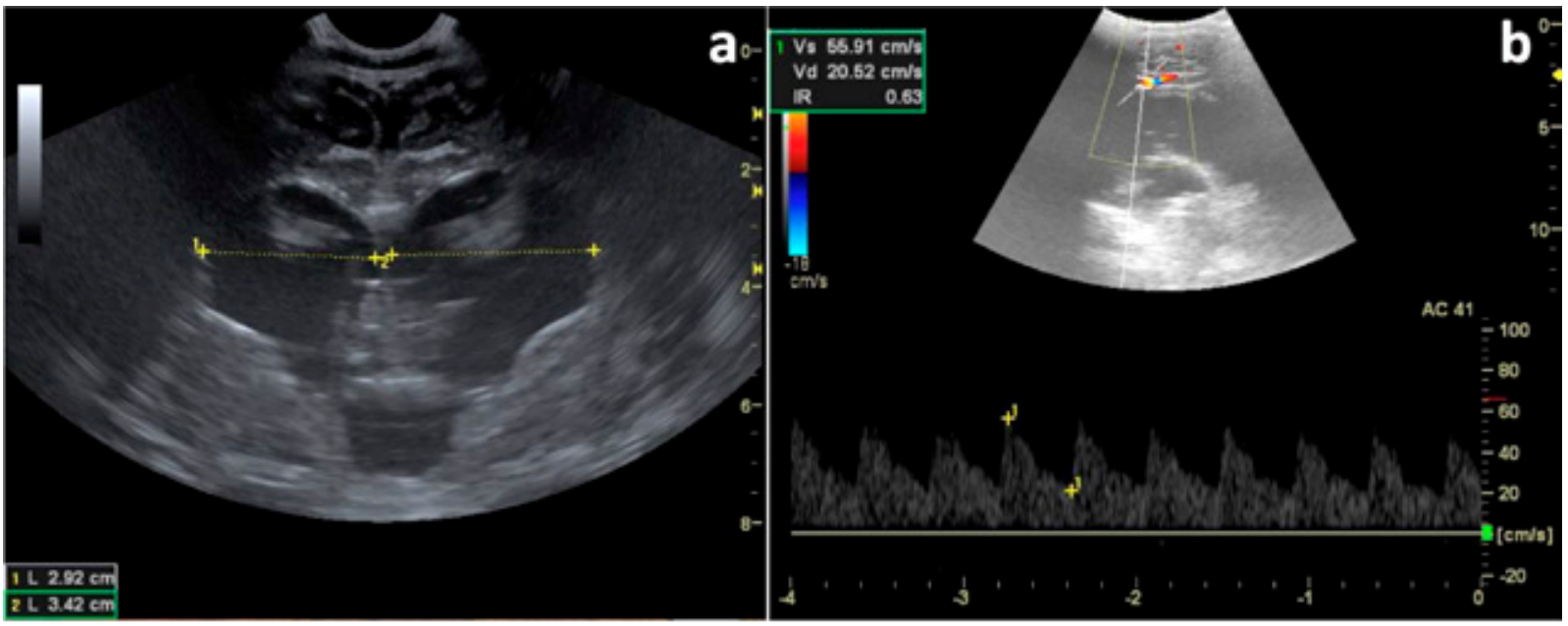

a) Visión frontal de corte coronal a nivel del agujero de Monro (índice ventricular de Levene).

b) Duplex con visión lateral de corte sagital a nivel de la línea media (imagen superior) y cuantificación del índice de resistencia de Pourcelot (IR) mediante doppler pulsado (imagen inferior). 


\section{DISCUSIÓN}

El QBB fue descrito por primera vez en 1900 por Joseph Blake. ${ }^{6}$ Es un divertículo quístico derivado del epéndimo que se forma, durante el desarrollo embrionario, del $4^{\circ}$ ventrículo. Entre un tercio y la mitad de los casos muestran su resolución espontánea como resultado de la fenestración tardía del foramen de Magendie, ${ }^{7,8}$ lo que da lugar al citado ventrículo y la cisterna magna. Se caracteriza, por tanto, por la existencia de un quiste infracerebeloso, ausencia de comunicación entre el cuarto ventrículo y el espacio subaracnoideo, e hidrocefalia tetraventricular. ${ }^{5}$ El vermis está bien desarrollado con una leve rotación en sentido antihorario. ${ }^{8}$

Se cree que el QBB pertenece al complejo sindrómico Dandy-Walker. ${ }^{6}$ Se ha descrito su asociación, en ocasiones, a otro tipo de alteraciones congénitas. ${ }^{9,10}$ Las manifestaciones clínicas de su persistencia no son muy bien conocidas. ${ }^{5}$ Varían desde el paciente asintomático a la presentación precoz de hidrocefalia y formas tardías en los adultos.

Aunque hay casos descritos en la literatura de QBB de presentación clínica atípica, ${ }^{5}$ la tortícolis como forma de presentación en lactantes pequeños no ha sido revelada hasta la actualidad. Este tipo de presentación es infrecuente, puesto que las fontanelas y las suturas en los lactantes de escasos meses de edad están aún bastante permeables. En este sentido, la presión intracraneal ocasionada debe ser muy elevada para llegar a producir un cuadro de tortícolis por episodios de enclavamiento intermitente.

En este caso, tampoco se habían revelado datos ecográficos prenatales sugerentes de la existencia de QBB o similares. Por tanto, presumiendo que se ha realizado una buena práctica asistencial, cabe plantearse la posibilidad de que haya habido una obstrucción posterior del foramen de Magendie que diera lugar al desarrollo del QBB al final de la gestación y/o en los primeros meses de la vida de la paciente. En su caso, se estaría describiendo un nuevo mecanismo fisiopatológico para el desarrollo del QBB.

Por último, cabe señalar que, ante un cuadro de tortícolis afebril en los lactantes pequeños, conviene incluir en el diagnóstico diferencial las malformaciones congénitas de la FCP, entre las que se encuentra el QBB. En muchos casos, como el descrito, la utilización de la ecografía cerebral transfontanelar en la consulta de pediatría de $\mathrm{AP}$, como una herramienta más de ayuda al diagnóstico, puede llegar a ser determinante. En otros muchos contextos clínicos, la disponibilidad de la ecografía a pie de cama ${ }^{8}$ en la AP de Salud puede contribuir también a mejorar la calidad asistencial en términos de eficacia y eficiencia, y a simplificar los protocolos de asistencia pediátrica con menor grado de iatrogenia.

\section{REFERENCIAS}

1. GötzeM, Hagmann S. Der Schiefhals beim Kind. Orthopade. 2019; 48(6):503-7.

2. BallockRT, SongKM. The prevalence of nonmuscularcauses of torticollis in children. J Pediatr Orthop. 1996; 16(4):500-4.

3. Herman MJ. Torticollis in infants and children: common and unusual causes. Instr Course Lect. 2006; 55:647-53.

4. Mayordomo-Colunga J, González-Cortés R, Bravo-Laguna MC, Martínez-Mas R, et al. Ecografía a pie de cama: ¿es el momento de incluirla en la formación del pediatra? An Pediatr (Barc). 2019; 91(3):206.e1-13.

5. Bontognali M, Poretti A, Guzman R, Huisman TA, et al. Blake's pouch cyst in children: Atypical clinical presentation. Neuroradiol J. 2018; 31(4):430-3.

6. Azab WA, Shohoud SA, Elmansoury TM, Salaheddin W, et al. Blake's pouch cyst. Surg Neurol Int. 2014; 5:112.

7. Ramaswamy S, Rangasami, R, Suresh S, Suresh I. Spontaneous resolution of Blake's pouch cyst. Radiol Case Rep. 2013; 8(4):877.

8. Vakakmudi UB, Rangasami R, Gopinath VN. Prenatal Blake pouch cyst with hydrocephalus. Neurol India. 2016; 64(4):830-1.

9. Myers KA, Wallis MJ, Fitt GJ, Sarnat HB, et al. Blake's pouch cyst in $13 q$ deletion syndrome: Posterior fossa malformations may occur due to disruption of multiple genes. Am J Med Genet A. 2017; 173(9):2442-5.

10. Shohoud SA, Azab WA, Alsheikh TM, Hegazy RM. Blake's pouch cyst and Werdnig-Hoffmann disease: Report of a new association and review of the literature. Surg Neurol Int. 2014; 5(Suppl 4):S282-8. 